\title{
Grahps of stable plane-Gauss maps
}

\author{
Catarina Mendes de Jesus ${ }^{1}$ \\ DM/UFJF, Juiz de Fora, MG, Brazil \\ Laércio José dos Santos ${ }^{2}$ \\ DM/UFJF, Juiz de Fora, MG, Brazil \\ Pantaleón D. Romero ${ }^{3}$ \\ DMFCT/CEU UCH,, València, Spain
}

\begin{abstract}
In this paper, we present immersions of a given closed and oriented surface $M$ in $\mathbb{R}^{3}$, where the two applications are stable: projection on the plane $x y$ and a Gauss map . The projection on the plane can be seen as a stable map of surfaces on the plane.
\end{abstract}

keywords: Closed sufarces, Graphs, stable maps

\section{Introduction}

The singular set of stable maps from closed surfaces $M$ to the plane consists of curves of double points, possibly containing isolated cusp points [4]. The apparent contour (i.e. the image of the singular set) is the number of immersed curves in $\mathbb{R}^{2}$ (possibly with cusps) whose self-intersections are all transverse and disjoint from the cusps. Graphs associated with the global study of stable applications of closed surfaces and oriented in the plane, was introduced in [6]. The singularities of a stable Gauss map, in Whitney's sense, being fold curves with isolated cusp points, are called the parabolic set of the surface $([1,2,9])$. Each parabolic curve in this set separates a hyperbolic region from an elliptic region of the surface. Graphs associated with the global study of stable Gauss applications of closed surfaces and oriented in the plane, was introduced in [7].

The purpose of this work is to present immersion constructions of closed and oriented surfaces in 3-space, with the coordinate system $(x, y, z)$, where the two maps are stable: projection on the plane $x y$, which can be seen as maps of surfaces in the plane, and the Gauss map. We show that any pair of trees can be realized as a graph of a pair of maps, one of them is a Gauss map and the other a map of the sphere in the plane.

\section{Stable maps}

Let $M$ and $N$ be smooth connected closed orientable surfaces and $f, g: M \rightarrow N$ be smooth maps between them. It is said that $f$ is $\mathcal{A}$-equivalent (or equivalent) to $g$ if there are orientationpreserving diffeomorphisms, $k: M \rightarrow M$ and $l: N \rightarrow N$, such that $g \circ k=l \circ f$. A smooth map $f: M \rightarrow N$ is said to be stable if all maps sufficiently close to $f$, in the Whitney $C^{\infty}$-topology (see [4]), are equivalent to $f$.

\footnotetext{
${ }^{1}$ cmendesjesus@ufjf.br

2laercio.santos@ice.ufjf.br

${ }^{3}$ pantaleon.romero@uchceu.es
} 
The concept of stability for a Gauss map of a surface immersed in $\mathbb{R}^{3}$ is slightly different from the general case of maps between surfaces in the sense that it depends on perturbations of the immersion rather than on those of the map itself.

Let $j: M \rightarrow \mathbb{R}^{3}$ be an immersion of $M$ in $\mathbb{R}^{3}$. Consider the following maps:

1. A stable projection $p_{v}: j(M) \rightarrow \mathcal{P} \subset \mathbb{R}^{3}$, where $\mathcal{P}$ is a orthogonal plane to $v \in \mathbb{R}^{3}$ and $p_{v}$ is the restriction to $j(M)$, of the projection of $\mathbb{R}^{3}$ in $\mathcal{P}$. A diffeomorphism $g: \mathcal{P} \rightarrow \mathbb{R}^{2}$ and $f_{2}: M \rightarrow \mathbb{R}^{2}$ given by $f_{2}=g \circ p_{v} \circ j$.

2. Gauss map $\mathcal{N}_{j}: j(M) \rightarrow \mathbb{S}^{2}$ of $j(M)$ and $f_{3}: M \rightarrow \mathbb{S}^{2}$ given by $f_{3}=\mathcal{N}_{j} \circ j$.

In this case, the map $f_{3}$ is called Gauss map of $M$, associated to the immersion $j$. The Gauss map $\mathcal{N}_{j}$ is said to be stable if there exists a neighborhood $\mathcal{U}_{j}$ of $j$ in the space $\mathcal{I}\left(M, \mathbb{R}^{3}\right)$ of immersions of $M$ into $\mathbb{R}^{3}$ such that for all $k \in \mathcal{U}_{j}$, the Gauss map $\mathcal{N}_{k}$ associated to $k$ is $\mathcal{A}$-equivalent to $\mathcal{N}_{j}$.

Definition 2.1. We say that the smooth map $f=\left(f_{2}, f_{3}\right): M \rightarrow \mathbb{R}^{2} \times \mathbb{S}^{2}$ is a stable plane-Gauss map if each $f_{i}, i=2,3$, is a stable map.

Let $f=\left(f_{2}, f_{3}\right): M \rightarrow \mathbb{R}^{2} \times \mathbb{S}^{2}$ be a stable plane-Gauss map. A point of the surface $M$ is a regular point of $f_{i},(i=2,3)$, if the map $f_{i}$ is a local diffeomorphism around that point and otherwise singular. We denote by $\Sigma f_{i}$ the singular set of $f_{i}$ and its image $B f_{i}=f_{i}\left(\Sigma f_{i}\right)$ is the branch set of $f_{i}$. We observe that regular points of $f_{3}$ corresponds, geometrically, to elliptic or hyperbolic points of $j(M)$. Singular points of $f_{3}$ corresponds to parabolic points of $j(M)$ (see [7]). By Whitney's Theorem (see [4]), $\Sigma f_{i}$ consists of closed curves with fold points, possibly containing isolated cusp points. Then the branch set of $f_{i}$, consists of a collection of closed curves immersed in the target surface with possible isolated cusps and self-intersections (double points). The orientation of the branch set is as follows: transverse a branch curve following the orientation with nearby points on our left that have two more inverse images than those on our right. The non-singular set (which is immersed in the target surface by the map $f_{i}$ ) consists of a finite number of regions.

\section{Graph of stable plane-Gauss maps}

The singular sets of two equivalent maps are equivalent, in the sense that, there is a diffeomorphism carrying one singular set onto the other and similarly for the branch sets. Thus any invariant diffeomorphism of singular sets or branch sets will automatically be a topological invariant of the map. Both the number of connected components of the singular set and the topological types of the regions are topological invariants. This information may be encoded on a weighted graph $\mathcal{G}^{i}\left(V^{i}, E^{i}, W^{i}\right)$, where the pair $\left(M, \Sigma f_{i}\right)$ may be reconstructed (up to diffeomorphism) (see [6,7]). On the weighted graph $\mathcal{G}^{i}\left(V^{i}, E^{i}, W^{i}\right)$ defined by a stable map $f_{i}$ each of its $E^{i}$ edges corresponds to a path-component of the singular set of $M$ and each of its $V^{i}$ vertices to a different regular region of the surface. An edge is incident to a vertex if and only if the corresponding singular curve to the edge lies in the boundary of the regular region corresponding to the vertex (see Figure 1). A weight is defined as the genus of the region that represents and it is attached to each vertex. The number $W^{i}$ is the sum of weights of all graph vertices. It will be called total weight of the graph. When $W^{i}=0$, we will denote the graph simply by $\mathcal{G}^{i}\left(V^{i}, E^{i}\right)(i=2,3)$.

It is remarkable that $V^{i}$ represents the number of connected components of $M \backslash \Sigma f_{i} ; E^{i}$ the number of connected components of $\Sigma f_{i}$ and $W^{i}$ the total sum of the genus of the components of $M \backslash \Sigma f_{i}$. Given orientations of the surfaces $M, \mathbb{R}^{2}$ and $\mathbb{S}^{2}$, a region of $M \backslash \Sigma f_{2}$ is positive if the map preserves orientation and negative otherwise. A region of $M \backslash \Sigma f_{3}$ is positive if it has positive Gaussian curvature and negative otherwise. A vertex of $\mathcal{G}^{i}\left(V^{i}, E^{i}, W^{i}\right.$ ) has a positive (or negative) label depending on whether the region that it represents is positive (or negative). Since 
each component of $\Sigma f_{i}$ is the boundary of a positive and a negative region, the signs of the vertices are assigned alternatively, hence the graph associated to stable map $f_{i}$ is bipartite. We denote by $V^{i \pm}$ the number of positive (negative) vertices and $W^{i \pm}$ the total weight associated to the positive (negative) vertices.

Figure 1 illustrates an embedding of the torus in the 3 -space, where $f_{2}$ is a map of the torus in the plane and $f_{3}$ is a Gauss map. Let us remark that the sets of singular curves of these maps $f_{2}$ and $f_{3}$ are not equivalent.

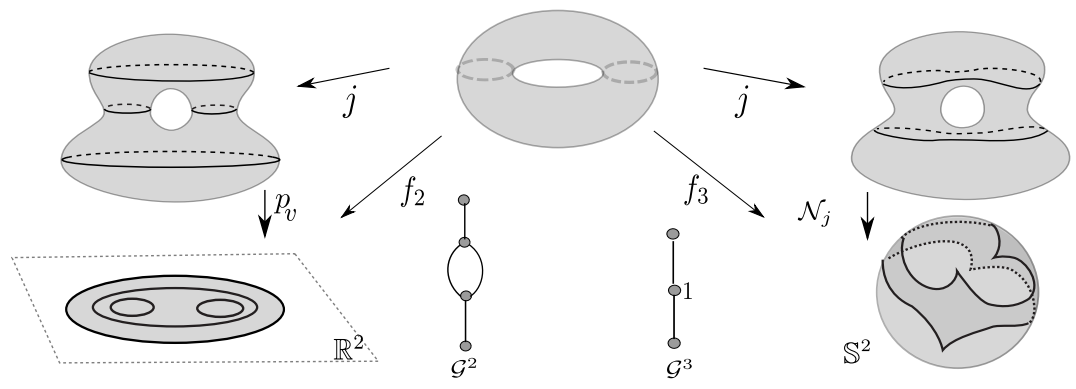

Figure 1: Example of stable plane-Gauss maps.

Definition 3.1. Let $\mathcal{G}$ be a connected graph with non-negative integer weights on its vertices. We say that $\mathcal{G}$ is a (see Figure 1):

1. Mendes-graph or simply $\mathcal{M}$-graph if exists a smooth connected closed surface $M$, a smooth surface $N$ and a stable map $f_{2}: M \rightarrow N$ such that $\mathcal{G}$ is the graph of $f_{2}$ (in the sense of [6]).

2. Fuster-graph or simply $\mathcal{F}$-graph if exists a smooth connected closed surface $M$ and an immersion $j: M \rightarrow \mathbb{R}^{3}$ whose stable Gauss map $f_{3}: M \rightarrow \mathbb{S}^{2}$, associated to $j$, has $\mathcal{G}$ as its associated graph (in the sense of [7]).

The Figure 1 illustrates the stable maps $f_{2}: \mathbb{S}^{2} \rightarrow \mathbb{R}^{2}$, whose $\mathcal{M}$-graph type $\mathcal{G}_{1}^{2}(4,6,0)$, and $f_{3}: \mathbb{S}^{2} \rightarrow \mathbb{S}^{2}$, whose $\mathcal{F}$-graph type $\mathcal{G}_{2}^{3}(3,2,1)$.

\subsection{Graph of stable plane-Gauss maps}

The graph $\mathcal{G}^{i}\left(V^{i}, E^{i}, W^{i}\right)$ is a global invariant and it classifies completely the topology of the stable maps regular set of $f_{i}$. Moreover, it will be used as a tool for the construction of examples of stable maps between surfaces.

The maps illustrates $f_{2}, g_{2}: \mathbb{S}^{2} \rightarrow \mathbb{R}^{2}$ in Figure 2 are non equivalent, where $j_{i}^{\prime} s$ indicates the respective embedding from $\mathbb{S}^{2}$ in $\mathbb{R}^{3}$ and $p_{v}, v=e_{3}$, the canonical projection. This example shows that the $\mathcal{M}$-graph is an invariants, because the apparent contour sets of $f_{2}$ and $g_{2}$ can not differ.
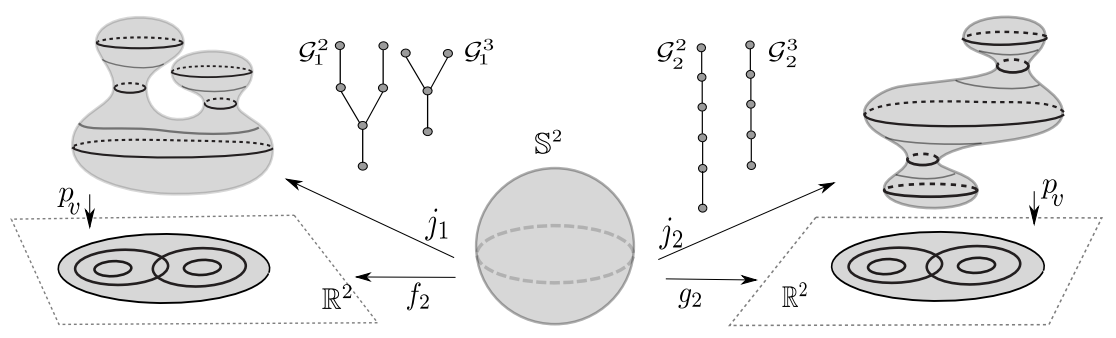

Figure 2: Examples of $\mathcal{M}$-graph and $\mathcal{F}$-graph type tree. 
Definition 3.2. Let $f=\left(f_{2}, f_{3}\right): M \rightarrow \mathbb{R}^{2} \times \mathbb{S}^{2}$ be a stable plane-Gauss map. We denote the graph of $f$ by $\left(\mathcal{G}^{2}, \mathcal{G}^{3}\right)$, where $\mathcal{G}^{2}$ is the graph of $f_{2}$ and $\mathcal{G}^{3}$ the graph of $f_{3}$.

Definition 3.3. We say that the pair of graphs $(\mathcal{G}, \mathcal{H})$ is a $\mathcal{M F}$-graph if exists a stable plane-Gauss map $f=\left(f_{2}, f_{3}\right): M \rightarrow \mathbb{R}^{2} \times \mathbb{S}^{2}$ such that the graph of $f_{2}$ is $\mathcal{G}$ the graph of $f_{3}$ is $\mathcal{H}$.

Given a closed and oriented surface $M$, by Definition 3.2, every stable plane-Gauss map $\left(f_{2}, f_{3}\right)$ : $M \rightarrow \mathbb{R}^{2} \times \mathbb{S}^{2}$ is associated with an $\mathcal{M F}$-graph. Some natural questions can be considered:

i) Is every graph $\mathcal{G H}$ an $\mathcal{M F}$-graph?

ii) Otherwise, what conditions should we impose on a graph $\mathcal{G H}$ in order to be an $\mathcal{M F}$-graph?

The motivation of the study on these pairs of graphs is find answers to these questions, among others. We will see some constructions of these maps that can lead to future answers to these questions in the general case.

\section{Lips and beaks transitions of plane-Gauss maps}

We can always obtain a new stable plane-Gauss map, associated with new $\mathcal{M} \mathcal{F}$-graphs by changing the immersion of $M$ in $\mathbb{R}^{3}$. These alterations can be made changing the Gauss maps $f_{3}: M \rightarrow \mathbb{S}^{2}$ and can change at the same time, or not, the image of the $f_{2}: M \rightarrow \mathbb{R}^{2}$, depending on the map in the plane or changing $f_{2}$ without varying $f_{3}$.

A codimension one transition corresponds to the intersection of the set $\Delta$, of non-stable maps, with generic isotopy from a given stable map $f_{i}$ to another stable map $g_{i}(i=2,3)$ that are in different path-components of $\mathcal{E}(M, N)$. In other words, this means that codimension one transition is the point at which a path between $f_{i}$ and $g_{i}$ transversely intersects a strata of $\Delta$. The types of transitions are described in [8], in the case of surfaces in the plane and the case of Gauss maps $([3,7])$. For lips and beaks transitions that changes the number of cusps of each $f_{i}$, the edge number of the graph, the number of vertices or the weight of the graphs, fulfills $g(M)=1-V^{i}+E^{i}+W^{i}$ $(i=2,3)$ is constant. For more details, see $[6,7]$.

Definition 4.1. A cusp point $x \in \Sigma f_{i}(i=2,3)$ is called positive (resp. negative) if its local mapping degree, in a neighborhood $U_{x}$ of $x$ is +1 (resp. - 1$)$ with respect to the given orientations.

i) Lips transition $\mathbf{L}_{i}^{ \pm}$of map $f_{i}(i=2,3)$ : increases the number of cusps and increases by 1 the number of singular curves of $f_{i}$ : $\mathbf{L}_{i}^{+}$increases by 1 the number of regions negative and $\mathbf{L}_{i}^{-}$ increases by 1 the number of regions positive. Consequently, $\mathbf{L}_{i}^{ \pm}$increases one vertex and one edge on the graph $\mathcal{G}^{i}$.

ii) Beaks transition $\mathbf{B}_{i}$ of map $f_{i}(i=2,3)$ : increases the number of cusps. Beaks transitions of $f_{i}$ can be classified in eight different transitions that change the graph $\mathcal{G}^{i}$ (see Figure 3 ):

$$
\begin{aligned}
& B_{v i}^{+, \pm} \text {: increases by } 1 \text { the number of vertices in } V^{i \pm} \text { and the number of edges } E^{i} . \\
& B_{v i}^{-, \pm} \text {: decreases by } 1 \text { the number of vertices in } V^{i \pm} \text { and the number of edges } E^{i} . \\
& B_{w i}^{+, \pm} \text {: increases by } 1 \text { the weight in } W^{i \pm} \text { and decreases by } 1 \text { the number of edges } E^{i} . \\
& B_{w i}^{-, \pm} \text {: decreases by } 1 \text { the weight in } W^{i \pm} \text { and increases by } 1 \text { the number of edges } E^{i} .
\end{aligned}
$$

A transition $\delta \in\left\{L_{i}^{ \pm}, B_{v i}^{ \pm, \pm}, B_{w i}^{ \pm, \pm}\right\}$that decreases the number of cusps, will be denoted by $-\delta$.

Figure 3 illustrates a sequence of transitions on the sphere that simultaneously change the $\mathcal{M}$-graph and the $\mathcal{F}$-graph. 
Remark 4.1. The beaks and lips transitions for maps in the plane, can change or not the $\mathcal{F}$ graph (see Figure 3). Similarly, beaks and lips for Gauss maps can change or not the $\mathcal{M}$-graph, as illustrates the Figure 4 .

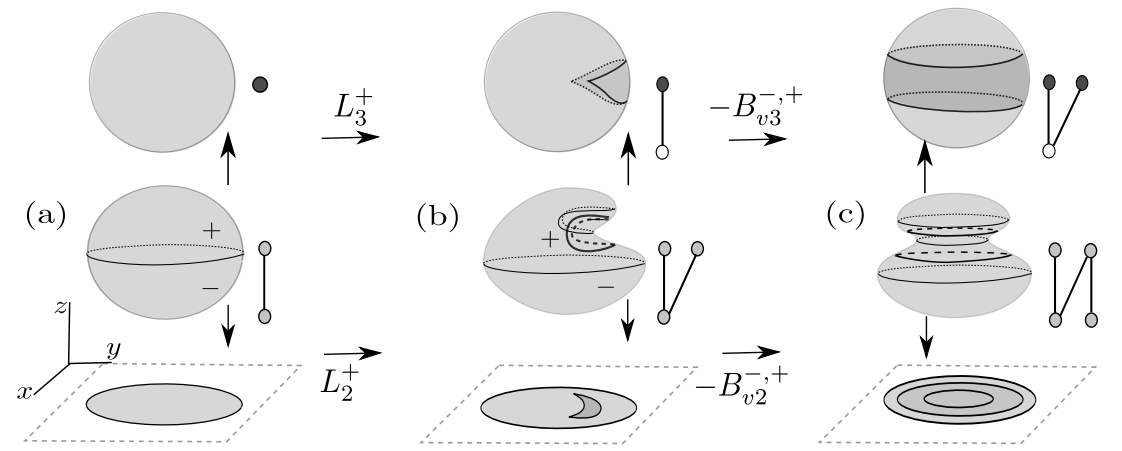

Figure 3: Transitions that change the $\mathcal{M}$-graph and $\mathcal{F}$-graph.
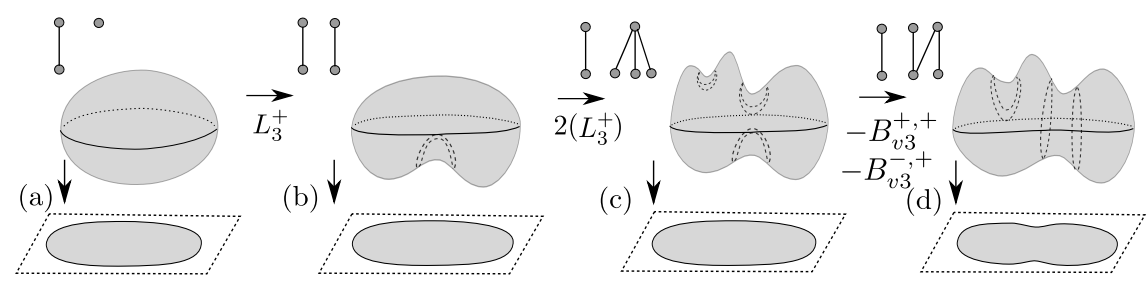

Figure 4: Transitions that only change the $\mathcal{F}$-graph.

Remark 4.2. Note that the sequence of transitions, in Figure 4, does not change the $\mathcal{M}$-graph it also does not change the apparent contour. This shows that the graph of the Gaussian map is an invariant that helps to refine the classification of maps in the plane.

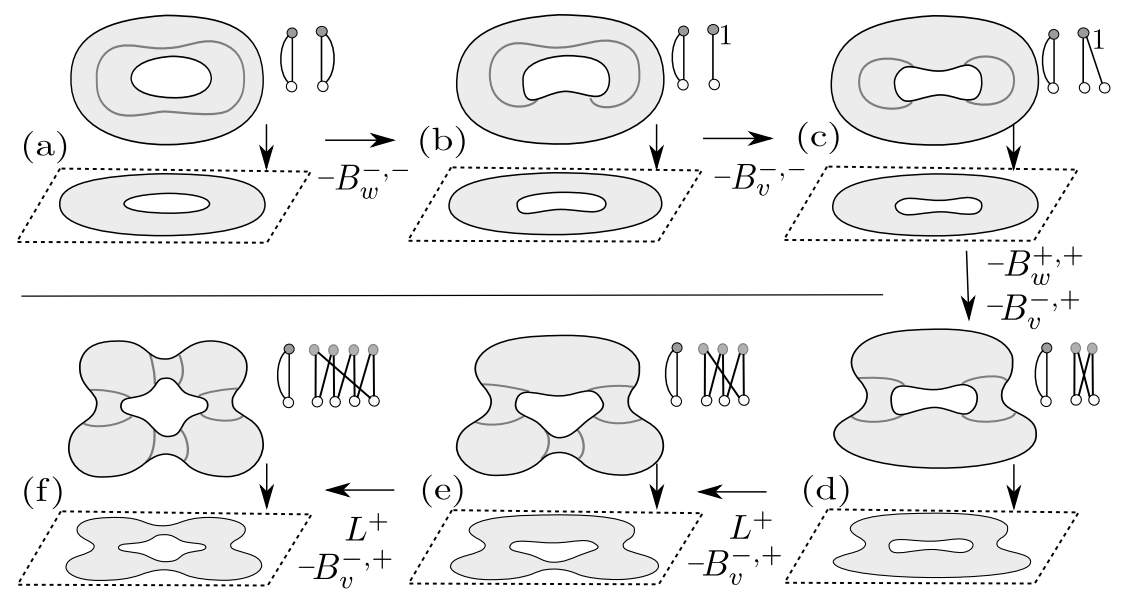

Figure 5: Example of $\mathcal{M F}$-graphs with cycles.

Figure 5 shows a sequence of stable maps of the torus with their graphs with transitions that changes the topology of parabolic curves keeping invariant the topology of map curves in the plane. In: (a) $\left(\mathcal{G}^{2}(2,2,0), \mathcal{G}^{3}(2,2,0)\right)$, each parabolic curve of $f_{3}$ has 4 cusp points: two positive 
and two negative (see [7]); (b) is obtained from (a) by transitions $-B_{w 3}^{-,-}$; it removes the pairs of positive cusps of $f_{3}$, leaving the new map with a elliptic region of genus one and one hyperbolic regions simply connected; (c) is obtained from (b) by transitions $-B_{v 3}^{-,-}$; dividing the parabolic curve and the hyperbolic region into two components; (d) is obtained from (c) by transitions $-B_{w 3}^{+,+}$, which decomposes a parabolic curve and removes the genus from the elliptic region and is followed by $-B_{v 3}^{-,+}$which decomposes the following parabolic curve, obtaining a map with 4 parabolic curves that separates two elliptic regions from two hyperbolic regions, all homeomorphic to the cylinder and with zero weight graph $\left(\mathcal{G}^{2}(2,2,0), \mathcal{G}^{3}(4,4,0)\right) ;(\mathrm{e})$ is obtained from $(\mathrm{d})$ by transitions $L_{3}^{+}$followed by $-B_{v 3}^{-,+}$, creating a new elliptic and hyperbolic region with the graph $\left(\mathcal{G}^{2}(2,2,0), \mathcal{G}^{3}(6,6,0)\right)$; the same happens with the transitions from (e) to (f) that realizes the graph $\left(\mathcal{G}^{2}(2,2,0), \mathcal{G}^{3}(8,8,0)\right)$. We can obtain a stable map that realizes the graph $\left(\mathcal{G}^{2}(2,2,0), \mathcal{G}^{3}(V, V, 0)\right)$, without changing the $\mathcal{M}$-graph in the following way (see Figure 5 -(e) and (f)).

\section{Realization of some trees with total weight zero}

In this section we present some families of graphs $\mathcal{G}^{2} \mathcal{G}^{3}$ that are $\mathcal{M} \mathcal{F}$-graphs, that is, graphs that can be realized by stable plane-Gauss maps in the sense that there is a smooth connected closed orientable surface $M$ and a stable plane-Gauss map $f=\left(f_{2}, f_{3}\right): M \rightarrow \mathbb{R}^{2} \times \mathbb{S}^{2}$ such that the graph of $f$ is $\left(\mathcal{G}^{2}, \mathcal{G}^{3}\right)$. This will be done through beaks transitions and lips on smaller maps already known. The results here will only be valid for zero weight graphs. For weight greater than zero will be treated in later works.

Before introducing the general result tree-type graphs (which satisfies $V=E+1$ ) with zero weight and some graphs with cycles in general, we will see some examples of realization. $\left(\mathcal{G}^{2}(2,1,0), \mathcal{G}^{3}(1,0,0)\right)$ can be realized (see Figure 4 -(a)) by a $\mathbb{S}^{2}$ embedding in the 3 -space with all elliptic points, which projected in a plane has a unique singular curve, which separates two regular regions. $\left(\mathcal{G}^{2}(2,1,0), \mathcal{G}^{3}(2,1,0)\right)$ can be realized as Figure 4 -(b), by lip transition $L_{3}^{+}$on (a) on one of the regular regions $f_{2}$, without changing $\Sigma f_{2}$.

Definition 5.1. The degree of a vertex $v$ in a graph is the number of edges incident to it. A tree it is called star positive (negative) if it has $V-1$ negative vertices (positive) with degree 1.

Theorem 5.1. The pair of graphs $\left(\mathcal{G}^{2}(2,1,0), \mathcal{G}^{3}(V, V-1,0)\right)$ is an $\mathcal{M F}$-graph, for all $V>0$.

Proof. We have shown that $\left(\mathcal{G}^{2}(2,1,0), \mathcal{G}^{3}(1,0,0)\right)$ is an $\mathcal{M F}$-graph. To realize the couple $\mathcal{G}^{2}(2,1,0) \mathcal{G}^{3}(V, V-$ $1,0)$ remove a negative (or positive) vertex, of degree 1 , from $\mathcal{G}^{3}(V, V-1,0)$ and suppose the pair $(\mathcal{G}(2,1,0), \mathcal{G}(V-1, V-2,0))$ realizes by a map $\left(g_{2}, g_{3}\right): \mathbb{S}^{2} \longrightarrow \mathbb{R}^{2} \times \mathbb{S}^{2}$. Through the lips transition $L_{3}^{+}$(or $\left.L_{3}^{-}\right)$on $\left(g_{2}, g_{3}\right)$, without changing the $\Sigma f_{2}$ (Remark 4.1 ), we can get a map $\left(f_{2}, f_{3}\right)$ which realizes the pair with $\mathcal{G}^{3}(V, V-1,0)$.

Corollary 5.1. The pair of graphs $\left(\mathcal{G}^{2}(2,1,0), \mathcal{G}^{3}(V, V-1,0)\right)$, where $\mathcal{G}^{3}$ is a star with $V>0$, is an $\mathcal{M F}$-graph.

Theorem 5.2. Every pair of graphs $\left(\mathcal{G}^{2}(V, V-1,0), \mathcal{G}^{3}(2,1,0)\right)$, with $V>1$, is an $\mathcal{M F}$-graph.

Proof. We have shown that $\left(\mathcal{G}^{2}(2,1,0), \mathcal{G}^{3}(2,1,0)\right)$ is an $\mathcal{M} \mathcal{F}$-graph. To realize the couple $\left(\mathcal{G}^{2}(V, V-\right.$ $\left.1,0), \mathcal{G}^{3}(2,1,0)\right)$ remove a negative (or positive) vertex, of degree 1 , from $\mathcal{G}^{2}(V, V-1,0)$ and suppose the pair $\left(\mathcal{G}^{2}(V-1, V-2,0), \mathcal{G}^{3}(2,1,0)\right)$ realizes by a map $\left(g_{2}, g_{3}\right): \mathbb{S}^{2} \longrightarrow \mathbb{R}^{2} \times \mathbb{S}^{2}$. Through the lips transition $L_{2}^{+}$(or $\left.L_{2}^{-}\right)$on $\left(g_{2}, g_{3}\right)$, without changing the $\Sigma f_{3}$, we can get a map $\left(f_{2}, f_{3}\right)$ which realizes the pair with $\mathcal{G}^{2}(V, V-1,0)$.

Corollary 5.2. The pair of graphs $\left(\mathcal{G}^{2}(V, V-1,0), \mathcal{G}^{3}(2,1,0)\right)$, where $\mathcal{G}^{2}$ is a star with $V>1$, is an $\mathcal{M F}$-graph. 
Theorem 5.3. All graph $\left(\mathcal{G}^{2}\left(V^{2}, V^{2}-1,0\right), \mathcal{G}^{3}\left(V^{3}, V^{3}-1,0\right)\right)$, with $V^{2}, V^{3}>1$, is an $\mathcal{M F}$-graph.

Proof. $\left(\mathcal{G}^{2}(2,1,0), \mathcal{G}^{3}\left(V^{3}, V^{3}-1,0\right)\right)$ can be realized by a map $\left(f_{2}^{1}, f_{3}^{1}\right)$ as Theorem 5.1. Then, chooses the hyperbolic and elliptical regions corresponding to vertices the $u_{i}$ and $v_{i}$ in the neighborhood of the parabolic curve associated with edges $u_{i} v_{i}$, of $\mathcal{G}^{3}\left(V^{3}, V^{3}-1,0\right)$. Lips transitions can be applied to one of the regular regions of $\left(f_{2}^{1}, f_{3}^{1}\right)$, as in Theorem 5.2 , properly, to obtain a map $\left(f_{2}, f_{3}\right)$ that realizes the graph $\left(\mathcal{G}^{2}\left(V^{2}, V^{2}-1,0\right), \mathcal{G}^{3}\left(V^{3}, V^{3}-1,0\right)\right)$, without changing the topology of the singular set of $f_{3}$.

\section{Conclusions}

All pair of graphs $\left(\mathcal{G}^{2}\left(V^{2}, V^{2}-1,0\right), \mathcal{G}^{3}\left(V^{3}, V^{3}-1,0\right)\right)$, with $V^{2}, V^{3}>1$, can be associated to an immersion $j: \mathbb{S}^{2} \rightarrow \mathbb{R}^{3}$, where are stable the projection $p_{v}: j\left(\mathbb{S}^{2}\right) \rightarrow \mathcal{P} \subset \mathbb{R}^{3}(\mathcal{P}$ is a orthogonal plane to $v \in \mathbb{R}^{3}$ and $p_{v}$ is the restriction to $j\left(\mathbb{S}^{2}\right)$, of the projection of $\mathbb{R}^{3}$ in $\mathcal{P}$ ) and the Gauss map $\mathcal{N}_{j}: j\left(\mathbb{S}^{2}\right) \rightarrow \mathbb{S}^{2}$ of $j\left(\mathbb{S}^{2}\right)$.

\section{References}

[1] Arnol'd, V. I., Gusein-Zade, S. M. and Varchenko, A. N. Singularities of differentiable maps. Vol. I. The classification of critical points, caustics and wave fronts, Translated from the Russian by Ian Porteous and Mark Reynolds, Monographs in Mathematics, 82. Birkhäuser Boston, Inc., Boston, MA, 1985.

[2] Banchoff, T., Gaffney, T. and McCrory, C. Cusps of Gauss Mappings, Pitman Books Limited, London, 1982.

[3] Bruce,J. W., Giblin, P. J. and Tari, F. Families of surfaces: height functions, Gauss maps and duals, Real and Complex Singularities, ed. W. L. Marar, Pitman Research Notes in Mathematics, volume 333, pages 148-178, 1995.

[4] Golubitsky, M. and Guillemin, V. Stable Mappings and Their Singularities, Springer Verlag, Berlin, 1976.

[5] Hacon, D., Mendes de Jesus, C. and Romero-Fuster, M.C. Topological invariants of stable maps from a surface to the plane from a global viewpoint, Real and Complex Singularities, in: Lecture Notes in Pure and Appl. Math, Dekker, New York, volume 232, pages 227-235, 2003. DOI:10.1201/9780203912089.

[6] Hacon, D., Mendes de Jesus, C. and Romero-Fuster, M.C. Stable maps from surfaces to the plane with prescribed branching data, Topology and Its Appl., volume 154, pages 166-175, 2007. DOI: $10.1016 /$ j.topol.2006.04.005.

[7] Mendes de Jesus, C., Moraes, S. M. and Romero-Fuster, M.C. Stable Gauss maps on surfaces from a global viewpoint, Bulletin of the Brazilian Mathematical Society, volume 42, pages 87-103, 2011. DOI: 10.1007/s00574-011-0005-8.

[8] Ohmoto, T. and Aicardi, F. First order local invariants of apparent contours. Topology, volume 45, chapter 23, pages 27-45, 2006. DOI: 10.1016/j.top.2005.04.005.

[9] Romero-Fuster, M. C. Sphere stratifications and the Gauss map, Proceedings of the Royal Society of Edinburgh, volume 95, pages 115-136, 1983. DOI: 10.1017/S0308210500015821. 